\title{
Characterisation of patients with a complete biochemical response to ursodeoxycholic acid
}

\author{
R A Jorgensen, E R Dickson, A F Hofmann, S S Rossi, K D Lindor
}

\begin{abstract}
Ursodeoxycholic acid (UDCA) leads to biochemical and clinical improvement in many patients with primary biliary cirrhosis (PBC); although, the response is variable. This study compared UDCA treated patients with complete normalisation of biochemical functions to those without such improvement. Of the 65 patients receiving UDCA, $12(19 \%)$ showed normalisation of liver biochemical functions at two years. The remaining 53 patients showed a less complete response. Mean (SD) alkaline phosphatase and total serum bilirubin values were significantly lower at entry in the patients whose liver biochemistry tests normalised (912 (732) $U / 1 v 1417(1021) \mathrm{U} / \mathrm{h}, \mathrm{p}=0.003$, and 0.7 (12.1 (5.2) $\mu \mathrm{mol} / /$ v 38.9 (48.5) $\mu \mathrm{mol} / /$, $\mathbf{p}=0.0002$, respectively), and percentage of UDCA in biliary bile acid was higher $(56 \cdot 3$ $(9.5) \%$ v $38.3(21 \cdot 1) \%, p=0 \cdot 03)$. Patients with biochemically and histologically less severe disease, and greater enrichment of biliary bile with UDCA, are more likely to respond favourably to the drug. The main objective of continued study will be to find out if normal liver biochemical functions can retard disease progression. The association of greater UDCA enrichment with complete biochemical responses suggests that higher doses of UDCA should be evaluated.

(Gut 1995; 36: 935-938)
\end{abstract}

Keywords: ursodeoxycholic acid, primary biliary cirrhosis.

Primary biliary cirrhosis (PBC) is a chronic cholestatic liver disease that characteristically progresses to cirrhosis and death from liver failure. ${ }^{1}$ Substantial improvements in biochemical or histological parameters, or both rarely, if ever, occur in the absence of drug treatment. A number of drugs have been evaluated in the treatment of this disease over the years in the context of clinical trials including D-penicillamine, colchicine, azathioprine, corticosteroids, chlorambucil, and cyclosporin. Improvements have occasionally been reported in biochemical functions, and even in histology or clinical symptoms, or both but seldom without substantial toxicity. Recently, ursodeoxycholic acid (UDCA) has been shown to be of benefit in the treatment of this disease and is virtually free of side effects. ${ }^{2}$

UDCA was proposed as a therapeutic approach to $\mathrm{PBC}$ on the basis that endogenous hydrophobic bile acids accumulate in chronic cholestatic disease and can result in hepatocyte damage. ${ }^{2}$ This theory is controversial, however, as several authors have described a change to a more hydrophilic acid composition in cholestatic liver disease. ${ }^{3}$ UDCA administration increases the concentrations of hydrophilic bile acids and prevents the hepatotoxic effects of other bile acids. ${ }^{4}$ In addition, it has recently been found to reduce HLA antigen expression on bile duct cells and hepatocytes. ${ }^{6}$ Biochemical improvements seen in patients treated with UDCA may be the result of these effects. In controlled and uncontrolled clinical trials, UDCA has been shown to improve biochemical tests of liver function and in two of the studies, improvement of liver histology was noted. ${ }^{7-9}$ In addition, the lack of associated major toxicity makes it the current treatment of choice among many physicians for patients with PBC.

During 1988 we began a randomised, double blind trial evaluating UDCA in the treatment of PBC in which we concluded that UDCA is an effective therapy for patients with PBC. UDCA was associated with delayed progression of the disease and lack of side effects. As part of our analysis a subset of patients was found who completely normalised their liver biochemical functions during two years of treatment. This finding led us to further evaluate the characteristics that distinguished these patients from those with a less complete biochemical response.

\section{Methods}

The patients described were enrolled in a randomised, double blind controlled trial designed to evaluate UDCA in the treatment of PBC. One hundred and eighty patients were entered with 89 patients randomised to receive UDCA and 91 to receive placebo. Sixty five of the patients treated with UDCA and 53 of the placebo treated patients had at least two years of follow up. All patients met criteria for the diagnosis of PBC including chronic cholestatic liver disease of at least six months' duration, an increase in alkaline phosphatase activity of at least 1.5 times the upper limit of normal, positive antimitochondrial antibody, biliary obstruction excluded by ultrasonography, computed tomography, or cholangiography, and liver biopsy done in the preceding three months compatible with the diagnosis of PBC. Exclusion criteria included previous treatment with UDCA, colchicine, corticosteroids, azathioprine, cyclosporin, chlorambucil, or D-penicillamine in the preceding three months 
TABLE I Entry characteristics

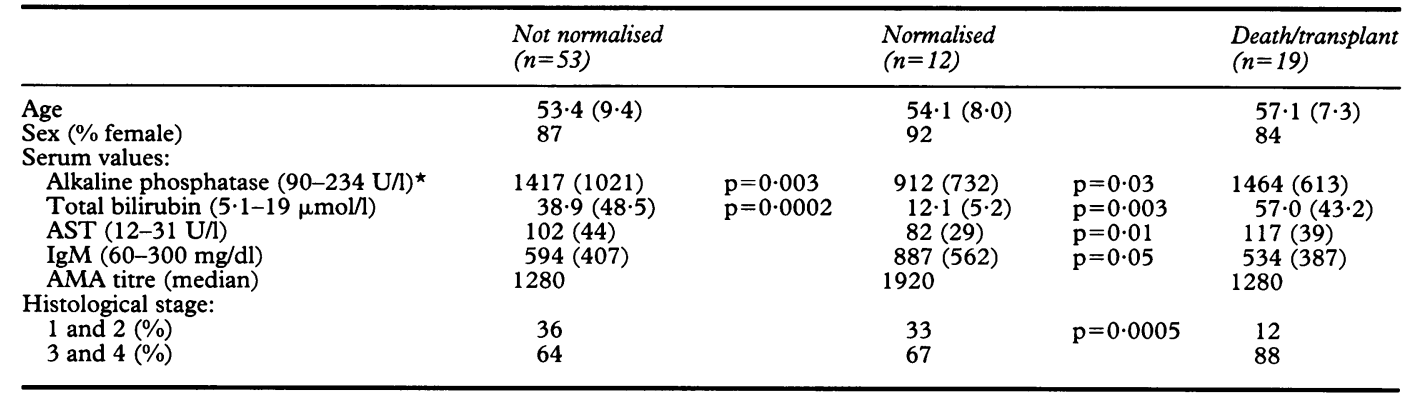

^Normal range given is that of a 51-55 year old woman. Normal range varies according to age and sex.

before study entry, anticipated need for transplantation within one year, recurrent variceal bleeds, spontaneous encephalopathy, or resistant ascites, pregnancy, age $<18$ years, or features suggestive of liver disease of other cause such as chronic alcoholic liver disease, chronic hepatitis B infection, or chronic active hepatitis. The study was designed to compare the treatment groups with respect to time to treatment failure. Treatment failure was defined as development of oesophageal varices or ascites in those without it at entry, worsening of fatigue or pruritus by two grades, doubling of total serum bilirubin (second value $>25.9 \mu \mathrm{mol} / \mathrm{l})$, histological progression by two stages or to cirrhosis, voluntary discontinuation of or inability to tolerate the drug treatment for any reason, transplantation or death.

Histological staging followed the Ludwig criteria $^{10}$ in which stage 1 was classified as portal hepatitis, stage 2 as periportal hepatitis, stage 3 as bridging necrosis or fibrosis, or both, and stage 4 as cirrhosis.

Severity of symptoms was assessed on the basis of a grading system. For pruritus grade 0 was no pruritus, grade 1 was mild pruritus, grade 2 was moderate pruritus including some interference with sleep, and grade 3 was severe pruritus with excoriations and substantial sleep disturbance. For fatigue grade 0 was no fatigue, grade 1 was mild fatigue that did not interfere with activity, grade 2 was moderate fatigue that requires extra rest, limits activity, but still able to work, and grade 3 was severe fatigue that meant the patient was unable to work.

A complete history, physical examination, and blood testing (including serum biochemical tests) were done on all patients at entry and yearly intervals. Liver biochemical functions were also measured every three months by mailed specimens. Endoscopy, liver biopsy, and ultrasound were done at entry and at two years. Bile samples were obtained during upper endoscopy to measure UDCA composition, which was performed using high pressure liquid chromatography. ${ }^{11}$

TABLE II Changes at two years

\begin{tabular}{|c|c|c|c|}
\hline & $\begin{array}{l}\text { Normalised } \\
(n=12)\end{array}$ & $\begin{array}{l}\text { Not normalised } \\
(n=53)\end{array}$ & \\
\hline $\begin{array}{l}\text { AST (U/l) } \\
\text { Alkaline phosphatase (U/l) } \\
\text { Total bilirubin }(\mu \mathrm{mol} / \mathrm{l}) \\
\text { IgM (mg/dl) } \\
\text { Biliary bile acid measureme }\end{array}$ & $\begin{array}{l}-54(30) \\
-687(727) \\
-3 \cdot 45(4 \cdot 32) \\
-457(502)\end{array}$ & $\begin{array}{l}-27(34) \\
-737(710) \\
-13 \cdot 0(36 \cdot 5) \\
-35(283)\end{array}$ & $\begin{array}{l}p=0.02 \\
p=N S \\
p=N S \\
p=0.02\end{array}$ \\
\hline \multicolumn{3}{|c|}{$\begin{array}{l}\text { Biliary bile acid measurements (\% of total bile acids): } \\
\text { UDCA }\end{array}$} & $\mathrm{p}=0.03$ \\
\hline
\end{tabular}

Patients were assigned to receive either UDCA or placebo. The treatment was given with meals and a bedtime snack three to four times per day, depending on the patient's weight, administering the $250 \mathrm{mg}$ tablets in a daily dose of $13-15 \mathrm{mg} / \mathrm{kg} /$ day. Patients, nurses, and physicians were blinded as to whether the patient was assigned to UDCA or placebo.

\section{Statistics}

Data were entered and stored using the Clinfo software package. Student's $t$ test was used for normally distributed paired samples and the Wilcoxon rank sum was used for nonparametric data.

\section{Results}

Of the 65 patients receiving UDCA who had two years of follow up, $12(19 \%)$ had normalised their values of alkaline phosphatase, aspartate aminotransferase (AST), and total serum bilirubin. The remaining 53 patients had shown reductions in their values of these enzymes but not to normal. At study entry these groups did not differ significantly in terms of age, sex, values of AST, AMA titre, IgM, or histological stage (Table I). The 12 patients who normalised had a significantly lower mean (SD) serum bilirubin concentration $(12.1(5.2) v 38.9(48.5), \mathrm{p}=0.0002)$ and a lower alkaline phosphatase activity (912 (732) $v 1417$ (1021), $\mathrm{p}=0.003$ ). The changes from baseline in liver biochemical tests at two years in the two groups of UDCA treated patients are shown (Table II). Those who normalised showed significant reductions in values of AST and IgM. Biliary bile acid measurements at two years showed a significantly higher percentage of UDCA after treatment in the total bile acids in the patients who normalised than those who did not $(56.3$ $(9 \cdot 5) \%$ v $38.3(21 \cdot 1) \%, \mathrm{p}=0.03)$. No correlation was found between initial total serum bilirubin concentrations and percentage of UDCA after treatment.

A total of 19 patients ( 7 receiving UDCA, 12 receiving placebo) died or were transplanted within the two year follow up period. When entry characteristics of these patients were compared with the 12 patients who normalised their biochemical functions, differences were noted in liver functions and histological stage. The patients who died or were 
transplanted presented with significantly higher values of alkaline phosphatase, AST, and total serum bilirubin. They were also histologically more advanced (Table I).

Changes over the initial three months from baseline alkaline phosphatase activities were compared between the group of 12 patients who normalised their liver function tests while receiving UDCA, the 7 patients who died or required a transplant while receiving UDCA, and the placebo treated patients. In the group of 12 patients there was a reduction in alkaline phosphatase activities of $84 \%$ towards the upper limit of normal (250 U/l). This compared with a reduction of $56 \%$ in the UDCA treated patients who died or required a transplant $(p=0.004)$, and an increase of $13 \%$ in the placebo treated patients $(\mathrm{p}=0.0001)$.

At two years, seven of 12 patients who normalised had remained stable histologically, four improved, and one patient progressed by one stage. Of the four patients who improved, three returned to stage 1 . Of the remaining 53 patients who did not normalise their liver function tests, 46 had liver histology available at two years. Of these 46 patients, $33(72 \%)$ stabilised or improved histologically and 13 progressed. Five of these 13 patients who progressed did so by two stages or to cirrhosis, which would define them as treatment failures. These differences were not significant, however.

One of the 12 patients in the group whose liver function tests normalised developed oesophageal varices. None of the 12 patients developed ascites as evaluated by ultrasound or clinical examination. Of the remaining group of 53 patients, five developed oesophageal varices and two patients developed ascites.

At two years changes in symptoms of pruritus and fatigue were not significantly different in the two groups of patients. In nine of the 12 who normalised $(82 \%)$, the degree of fatigue remained stable or lessened. The fatigue in 43 of the remaining 53 patients $(81 \%)$ remained the same or improved. Pruritus improved or remained the same in all 12 patients who normalised. Pruritus improved or remained stable in 44 of the 53 patients who did not normalise and worsened in nine of these patients.

None of the patients in the group who normalised died or required a liver transplant. Of the group of 53 patients who did not normalise, four patients died, three were transplanted, and one has been referred for a transplant.

One patient in the group of 12 patients who normalised while receiving UDCA, and 15 in the group of 53 patients who did not normalise with UDCA, progressed to treatment failure. This was not statistically significant.

In the group of 53 patients randomised to placebo, 29 progressed to treatment failure status. There was a significant difference when these numbers were compared with those progressing to treatment failure status in the groups who normalised and did not normalise while receiving UDCA $(p=0.002$ and 0.004 , respectively).

\section{Discussion}

UDCA has been shown to lead to biochemical improvements in patients with PBC. $27812 \mathrm{We}$ have found a subset of these patients that completely normalised their liver tests. At study entry these patients who normalised their liver tests did not differ from the remaining patients randomised to receive UDCA with respect to age, sex, histological stage, values of AMA, IgM, AST or in clinical symptoms. These patients may have represented a group with less severe disease although they overall were histologically similar. Values of alkaline phosphatase and total serum bilirubin were significantly lower in these patients at entry. Biochemical normalisation at two years could not be predicted from the response at three months. The percentage of UDCA in biliary bile was significantly higher in these patients after two years of treatment. It is possible that the increased concentrations of UDCA in bile are directly related to the improved response seen in this group of patients. Because all patients received the drug in a dose of 13 to 15 $\mathrm{mg} / \mathrm{kg} /$ day some patients may have better absorption or compliance, or both with the dose regimen. Efforts to further improve enrichment of bile with UDCA particularly in those patients who exhibited a less complete response should be assessed. Such approaches may entail increased doses of UDCA to increase bile acid enrichment or addition of other drugs to inhibit production of endogenous bile acid given along with UDCA. ${ }^{13} 14$

When the clinical course of these patients was compared with the patients who did not normalise their liver functions we found no differences with respect to changes in symptoms (pruritus or fatigue), histological progression, development of varices or ascites or progression to death or transplant. Longer follow up in more patients would be required to detect significant differences in these criteria, however.

We did find that 11 of 12 patients who normalised $(92 \%)$ either remained stable histologically or improved compared with $72 \%$ in the group who did not normalise. Three of the patients with normal liver tests who improved histologically returned to stage 1 and in two of these patients diagnostic abnormalities suggestive of PBC were not found at two years. None of the patients in the group who did not normalise returned to stage 1 . It would seem that there may be a trend toward corresponding histological improvement or remission in the patients who normalise although again longer follow up is probably required. Histological improvement has been suggested in other trials with UDCA. Leuschner et $a l^{7}$ showed improvement in hepatic histology in six of 10 patients receiving UDCA compared with only one of eight placebo patients. Poupon showed improvement in parameters of inflammation on liver biopsy in patients receiving UDCA compared with those receiving placebo at two years. ${ }^{2}$

The patients who normalised presented with lower initial concentrations of serum bilirubin, which would predict a better prognosis as bilirubin has been shown to be the most 
important prognostic indicator in this liver disease. ${ }^{15}$ It is possible that this finding is related to their improved response. Patients who died or were transplanted within the two year follow up period presented initially with more abnormal liver functions and were histologically more advanced than the 12 patients who responded with biochemical normalisation. These characteristics would predict a poorer prognosis and less favourable response to the drug. These findings support the idea that starting treatment earlier in the course of the disease when possible, is preferred (particularly as the drug is well tolerated and free of significant side effects).

The significant reduction seen in IgM concentrations at two years in those who showed a return to normal of their liver functions supports the view that $\operatorname{IgM}$ concentrations are valuable in monitoring the course of PBC. These findings are in agreement with those previously reported by Poupon ${ }^{2}$ and Wolfhagen ${ }^{16}$ and emphasise the potential role of UDCA in changing the immune response.

Biochemical, histological or clinical remissions in PBC patients treated with drugs other than UDCA are unusual. Often these drugs have limited benefit or are associated with significant side effects. Drugs such as D-penicillamine and colchicine have been evaluated. D-penicillamine in various clinical trials has been shown to be of no benefit in PBC. ${ }^{17-19}$ Colchicine did not have an effect on histological features although it did improve liver enzymes, ${ }^{2021}$ if only temporarily. Chlorambucil had little effect on liver functions and was associated with major toxicity. ${ }^{22}$ Cyclosporin has been associated with biochemical and histological remission but associated toxicity and the expense of monitoring drug concentrations made the drug less than ideal as a form of treatment. ${ }^{23}$

We have described a subset of patients treated with UDCA who have exhibited complete biochemical normalisation of liver function. Further study of patients with PBC who exhibit biochemical or histological remission, or both is warranted to better characterise this group. Methods to improve the degree of enrichment, such as higher doses of UDCA should be the subject of continued study and will help to further clarify the response in this subset of patients. The characteristics of patients most likely to exhibit biochemical normalisation should be delineated. Lastly, it needs to be discovered if these changes are associated with significant improvement in clinical and histological features as well as improved survival or delayed need for orthotopic liver transplantation.
Presented at Digestive Disease Week, Boston, MA, 15 May 1993.

1 Kaplan MM. Primary biliary cirrhosis. N Engl $\mathcal{F}$ Med 1987; 316: 521-8.

2 Poupon RE, Balkau B, Eschwege E, Poupon R. A multicenter, controlled trial of ursodiol for the treatment of primary biliary cirrhosis. $N$ Engl $7 \mathrm{Med} 1991$; 324: primary

3 Van Berge Henegouwen GP, Brandt $\mathrm{KH}$, Eyssen $\mathrm{H}$, Parmentier G. Sulfated and unsulfated bile acids in serum, bile, and urine of patients with cholestasis. Gut serum, bile, and

4 Stiehl A, Rudolph G, Raedsch R, Moller B, Hopf U, Stiehl A, Rudolph G, Raedsch R, Moller B, Hopf U,
Lotterer E, et al. Ursodeoxycholic acid-induced changes of plasma and urinary bile acids in patients with primary biliary cirrhosis. Hepatology 1990; 12: 492-7.

5 Galle PR, Theilmann L, Raedsch R, Otto G, Stiehl A. Ursodeoxycholate reduces hepatotoxicity of bile salts in primary human hepatocytes. Hepatology 1990; 12: 486-91.

6 Yoshikawa M, Tsujii T, Matsumura K, Yamao J, Matsumura Y, Kubo R, et al. Immunomodulatory effects of ursodeoxycholic acid on immune responses. Hepatology 1992; 16: 358-64.

7 Leuschner U, Fischer H, Kurtz W, Guldutuna S, Hubner $\mathrm{K}$, Hellstern A, et al. Ursodeoxycholic acid in primary biliary cirrhosis: results of a controlled double-blind trial biliary cirrhosis: results of a controlled
Gastroenterology 1989; 97: 1268-74.

8 Batta AK, Arora R, Salen G, Katz S. Ursodeoxycholic acid improves liver function and reduces serum and urinary endogenous bile acids in primary biliary cirrhosis. Hepatology 1988; 8: 1221

9 Hadziyannis S, Hadziyannis E. A randomized controlled trial of ursodeoxycholic acid in primary biliary cirrhosis (PBC) [Abstract]. Hepatology 1988; 8: 1421.

10 Ludwig J, Dickson ER, McDonald GSA. Staging of chronic nonsuppurative cholangitis (syndrome of primary biliary cirrhosis). Virchows Arch A 1978; 379: 103-12.

11 Rossi SS, Converse JL, Hofmann AF. High pressure liquid chromatographic analysis of conjugated bile acids in human bile: simultaneous resolution of sulfated and unsulfated lithocholyl amidates and the common conunsulfated lithocholyl amidates and the comm
jugated bile acids. F Lipid Res 1987; 28: 589-95.

12 Lindor KD, Dickson ER, Baldus WP, Jorgensen RA, Ludwig J, Murtaugh PA, et al. Ursodeoxycholic acid in the treatment of primary biliary cirrhosis. Gastroenterology 1994; 106: 1284-90.

13 Lillienau J, Schteingart CD, Hofman AF. Physiochemical and physiological properties of cholylsarcosine: a potential replacement detergent for bile acid deficiency states in the small intestine. 7 Clin Invest 1992; 89: 420-31.

14 Heuman DM, Vlahcevic ZR, Pandak WM, Hylemon PB, Kim YS, Lillienau J, et al. Effect of cholylsarcosine on hepatic cholesterol and bile acid synthesis and bile secretion in rats. Gastroenterology 1992; 103: 1641-8.

15 Dickson ER, Grambsch PM, Fleming TR, Fisher LD, Langworthy A. Prognosis of primary biliary cirrhosis: model for decision making. Hepatology 1989; 10: 1-7.

16 Wolfhagen FHJ, van Buuren HR, van Berge Henegouwen GP, van Hattum J, Eskens F, Kerbert-Dreteler MJ, et al. Can ursodeoxycholic acid monotherapy induce disease remission in primary biliary cirrhosis? Hepatology 1993; 18: $220 \mathrm{~A}$.

17 Dickson ER, Fleming TR, Wiesner RH, Baldus WP, Fleming CR, Ludwig J, et al. Trial of penicillamine in advanced primary biliary cirrhosis. $N$ Engl $f$ Med 1985 ; 312: $1011-5$.

18 Neuberger J, Christensen E, Portmann B, Caballeria J, Rodes J, Ranek L, et al. Double blind controlled trial of D-penicillamine in patients with primary biliary cirrhosis. Gut 1985; 26: 114-9.

19 Matloff DS, Alpert E, Resnick RH, Kaplan MM. A prospective trial of D-penicillamine in primary biliary cirrhosis. N Engl f Med 1982; 306: 319-26.

20 Bodenheimer HC Jr, Schaffner F, Pezullo J. Evaluation of colchicine therapy in primary biliary cirrhosis. Gastroenterology 1988; 96: 124-9.

21 Kaplan MM, Alling DW, Zimmerman HJ, Wolfe HJ, Sepersky RA, Hirsch GS, et al. A prospective trial of colchicine for primary biliary cirrhosis. $N$ Engl $\mathfrak{f} \mathrm{Med}$ 1986; 315: 1448-54.

22 Hoofnagle JH, Davis GL, Schafer DF, Peters M, Avigan MI, Pappas SC, et al. Randomized trial of chlorambucil for primary biliary cirrhosis. Gastroenterology 1986; 91: for primary

23 Wiesner RH, Ludwig J, Lindor KD, Jorgensen RA, Baldus WP, Homburger HA, et al. A controlled trial of cyclosporine in the treatment of primary biliary cirrhosis. N Engl f Med 1990; 322: 1419-24. 